\section{Fibrin glue inhibits migration of ocular surface epithelial cells}

AM Yeung, LA Faraj, OD Mclntosh, VK Dhillon and HS Dua

Eye (2016) 30, 1389-1394; doi:10.1038/eye.2016.127; published online 1 July 2016

\section{Purpose Fibrin glue has been used successfully in numerous ophthalmic surgical procedures. Recently, fibrin glue has been used in limbal stem cell transplantation to reduce both operative time and to negate the need for sutures. The aim of this study was to determine the effects of fibrin glue on epithelial cell migration in vitro.}

Methods Corneoscleral rims were split to retain the epithelial layer, Bowman's layer, and anterior stroma. Rims were cut into eight equal-sized pieces and were placed directly on culture plates or affixed with fibrin glue. Rims were maintained in culture for 25 days and epithelial cell growth was monitored. Cells were photographed to measure area or growth and immunofluorescence staining of explants for fibrin was performed.

Results Explants that were glued demonstrated significantly delayed epithelial cell growth and migration as compared with explants without glue. By day 16, all fibrin glue had dissolved and coincided with onset of cell growth from glued explants. Cell growth commenced between days 3 and 4 for control explants without glue and around days 14-16 for explants with fibrin glue. Conclusions Fibrin glue delays epithelial cell migration by acting as a physical barrier and can potentially interfere with explant-derived limbal epithelial cell migration on to the corneal surface. We propose that glue should be used to attach the conjunctival frill of the limbal explant but care should be taken to ensure that the glue does not wrap around the explant if used to secure the explant as well. Strategic use of glue, to attach the recessed conjunctiva, can be advantageous in delaying conjunctival cell migration and reducing the need for sequential sector conjunctival epitheliectomy.

\section{Introduction}

The corneoscleral limbus together with the palisades of Vogt are known to be repositories of corneal epithelial stem cells. ${ }^{1}$ Damage to the corneoscleral limbus by disease or injury can lead to limbal stem cell deficiency (LSCD). Conditions affecting the limbal stem cells can be congenital such as aniridia or acquired such as chemical or thermal injury or chronic inflammation as in Stevens Johnson syndrome. ${ }^{1,2}$

Symptoms of LSCD include reduced vision, tearing, blepharospasm, photophobia, and recurrent episodes of pain. Clinical signs of LSCD span a wide range from altered limbal anatomy, metaplastic conjunctivalised epithelium on the cornea, persistent epithelial defect to complete cover of the corneal surface with a fibrovascular membrane that may be associated with scarring and calcification. The hallmark of LSCD is the growth of conjunctival tissue over the region usually occupied by corneal epithelium that is known as conjunctivalisation $^{2}$ of the cornea. The diagnosis of LSCD is essentially clinical with delayed fluorescein staining of the conjunctivalised area. Confocal microscopy impression cytology and biopsy can provide confirmatory evidence.

LSCD is classified as partial or complete and unilateral or bilateral. Partial LSCD with visual axis involvement can be treated with mechanical debridement of the conjunctival epithelium known as sequential sector conjunctival epithelialectomy $(\mathrm{SSCE})^{3}$ wherein the abnormal epithelium is removed and the denuded area allowed to reepithelise from the surviving healthy limbal epithelium. Patients with total unilateral LSCD are ideally managed by
Larry A Donoso Laboratory for Eye Research, Section of Academic Ophthalmology, Division of Clinical Neuroscience, University of Nottingham, Nottingham, UK

Correspondence: HS Dua, Section of Academic Ophthalmology, B Floor, Eye ENT Centre, Queens Medical Centre, University Hospital, Derby Road, Nottingham NG7 2UH, UK

Tel: +44 (0)115 849 3354; Fax: +44 (0)1159709963. E-mail: Harminder.dua@ nottingham.ac.uk

Received: 6 January 2016 Accepted in revised form: 1 May 2016 Published online: 1 July 2016 
autolimbal transplantation. Bilateral total LSCD requires allolimbal transplantation from a living related donor or from a cadaver donor. For autolimbal and living related donor allolimbal transplants, traditionally, two clock hours of superior and inferior donor limbal tissue with a $3 \mathrm{~mm}$ frill of adjacent donor conjunctiva and $<1 \mathrm{~mm}$ of peripheral corneal tissue are excised and transplanted to the corresponding recipient sites after dissecting off the fibrovascular pannus. These explants are sutured in place, donor conjunctiva to the recipient conjunctiva posteriorly and the donor limbus to the recipient limbus anteriorly. ${ }^{4}$ More recently, there have been reports on use of fibrin glue in limbal transplantation for LSCD. ${ }^{5-8}$ The use of fibrin glue in this and other ophthalmic surgery is known to reduce both operative time and postoperative inflammation and pain. ${ }^{9-11}$

Our clinical experience with the use of fibrin glue to attach autologous and living related limbal grafts was that cell growth from the explants was delayed compared with use of sutures. This prompted us to undertake this study with the aim to determine in vitro the effects of fibrin glue on epithelial cell growth and migration from corneoscleral rims.

\section{Materials and methods}

\section{Tissue preparation and cell culture}

Five corneoscleral rims were obtained following penetrating keratoplasty from donor eyes that were retrieved with consent for research and transplantation. Corneoscleral discs were stored in organ culture medium (Eagle's minimum essential medium) for 3 to 4 weeks before transplantation. All rims were replaced in the culture medium and processed for culture within $72 \mathrm{~h}$ of transplantation. In brief, the excess sclera was trimmed and the posterior stroma, Descemet's membrane, and endothelium were removed. The rims were cut into 8-10 equal size pieces and set up as explants in tissue culture (CnT-20 Corneal Epithelial Medium (CELL n TEC Advanced Cell Systems AG, Bern, Switzerland), Gentamicin/Amphotericin $1 \mathrm{ml}$ (Life Technologies Ltd, Paisley, UK), and Plasmocin $1 \mathrm{ml}$ (InvivoGen, San Diego, CA, USA)). Explants were placed epithelial side up on Nunclon $35 \times 10 \mathrm{~mm}$ culture plates (VWR International Ltd, Leicestershire, UK). The explants were attached to the culture plate either with $50 \mu$ l fibrin glue ('Tisseel', Baxter Healthcare Corporation, Berkshire, UK) or made to adhere directly by air drying, without fibrin glue.

Explants were cultured in cornea epithelial culture medium that was replaced every 2-3 days. Explants were cultured for 21 days, with photographs taken on days 1, 7, and 16. They were then processed for immunofluorescence staining to detect fibrin.

\section{Light microscopy and growth rates}

Epithelial cell growth from explants was determined using a Nikon Eclipse TS 100 microscope ( $\times 40$ magnification; Kingston Upon Thames, UK). Each culture plate had a grid incorporated at the bottom with each square having an area of $4 \mathrm{~mm}^{2}$. The total number of squares covered by epithelial cells was recorded every $2-3$ days using a manual tally counter and these data in turn were expressed as a percentage of growth against time.

Each explant was removed from the culture dish and TrypLE (Gibco Invitrogen, Paisley, UK, cat. no. 12604-021) was added for $5 \mathrm{~min}$ to detach the cell sheet from the culture dish. The cell suspension was transferred to a universal tube (Sterilin Ltd, Newport, UK, cat. no. LS-M0570E) and the TrypLE was neutralised by the addition of culture medium. The tube was centrifuged for $5 \mathrm{~min}$ at $250 \mathrm{~g}$ and the cell pellet obtained was resuspended in $2 \mathrm{ml}$ of culture medium using gentle agitation of the tube. Then, $10 \mu 1$ of cell suspension was extracted with a pipette and placed in an Eppendorf tube with $10 \mu 1$ of Trypan blue (cat. no. T8154; Sigma, Poole, UK). The cell suspension was used to charge the haemocytometer counting chamber and the cells counted using the $10 \times$ objective. The number of viable cells (unstained by Trypan blue) in the area of 16 squares indicated by each of the 5 circles in the haemocytometer counting chamber were counted after 21 days.

\section{Immunofluorescence}

Explants were removed from culture plates at corresponding time points mentioned above and washed with phosphate-buffered saline (PBS). Next, they were placed in optimal cutting temperature compound (VWR International Ltd) and snap frozen in liquid nitrogen. Samples were kept in a $-80^{\circ} \mathrm{C}$ freezer until further processing. Tissue blocks were cut with a cryomicrotome (Lieca, Wetzlar, Germany) at a thickness of 6-7 $\mu \mathrm{m}$. Sections were fixed with $100 \%$ ice-cold acetone for $10 \mathrm{~min}$, washed with $1 \times$ PBS for $5 \mathrm{~min}$, and then blocked with $3 \%$ bovine serum albumin ( $3 \%$ BSA/1 $\times$ PBS) for $1 \mathrm{~h}$ at room temperature. Samples were incubated with primary antibody, anti-fibrin $\alpha$-chain (ab19079; dilution, $1: 200$; mouse; Abcam, Cambridge, UK) overnight at $4{ }^{\circ} \mathrm{C}$. The samples were washed with PBS and incubated with secondary antibody Alexa Fluor 488 (A-21202; dilution, 1 : 300; donkey anti-mouse; Life Technologies Ltd, Paisley, UK) for $1 \mathrm{~h}$. Samples were counterstained with 4',6diamidino-2-phenylindole (DAPI) and mounted with fluorescent mounting medium. Slides were examined under a fluorescent microscope (BX51, U-RFL-T, Olympus, Tokyo, Japan; Prior Proscan II, Prior Scientific Inc., 
Rockland, MA, USA). All research adhered to the tenets of the Declaration of Helsinki.

\section{Results}

\section{Light microscopy}

The first day after explants were placed (day 1), there was no evidence of growth from either fibrin glued explants or control explants. Between days 3 and 4, control explants began to demonstrate epithelial growth whereas fibrin explants did not. On day 7 (Figure 1a), some of the explants attached with fibrin glue demonstrated dissolution of glue at places along the margin. From these sites, evidence of epithelial growth could be seen. In contrast, explants where glue had persisted had no growth of epithelial cells. By days 14-16 (Figures 1b and 2) the glue had dissolved from all explants and epithelial cell growth was evident along the entire margin.
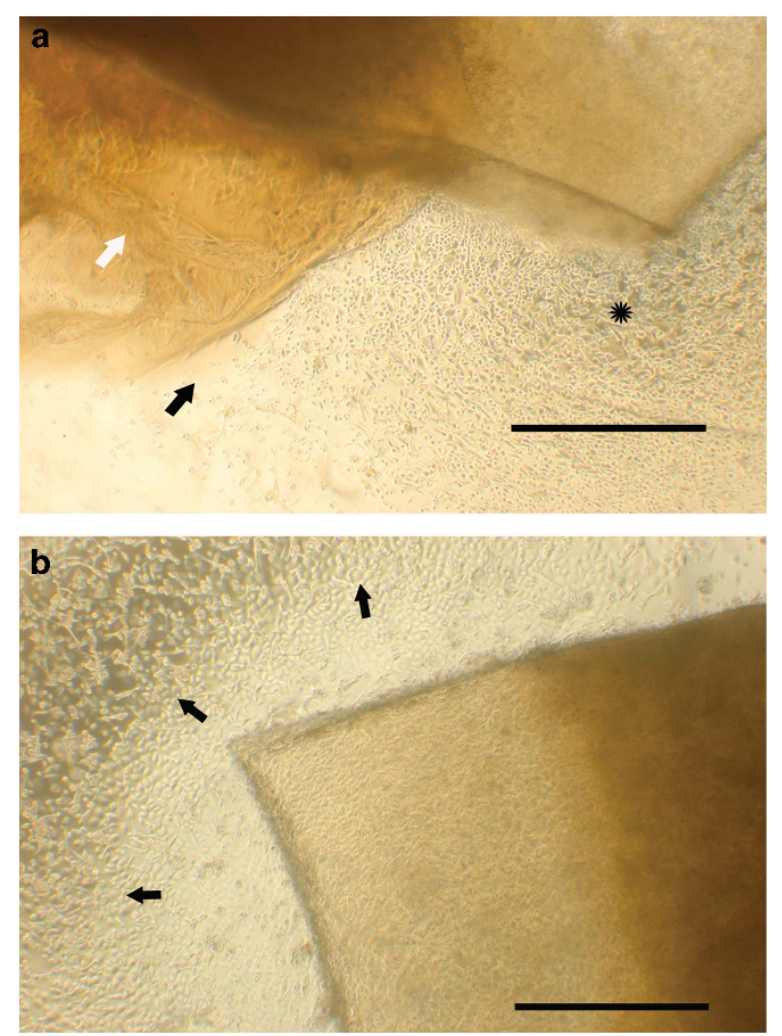

Figure 1 (a) Cell growth is visible from glued explants at sites where the glue has dissolved (star) compared with limited growth (black arrow) from sites where fibrin glue (white arrow) was still present (scale bar $=1000 \mu \mathrm{m}$ ). (b) Uniform cell growth is seen to occur from the margins of a glued explant, on day 16 following dissolution of the fibrin glue (scale bar $=1000 \mu \mathrm{m}$ ).

\section{Cell counts and cell growth}

Total epithelial cell growth, as determined by a haemocytometer, demonstrated that the mean number of cells after 21 days in the control group was 450000 $( \pm 122474)$ compared with $252500( \pm 60759)$ in the fibrin glue group and that there was a significant statistical difference in the number of cells between control compared with glue $(P=0.019)$.

Percentage area of epithelial cell growth as plotted against time is given in Figure 2. In control samples cell growth started on day 3 and expanded rapidly over the following 3 weeks to reach $100 \%$ confluence by day 24 . In comparison, cell growth started only on day 14 and after a phase of slow growth for about a week, cell growth picked up rapidly to match that of the control group. Fibrin glue thus retarded cell growth from explants for up to 2 weeks.

\section{Immunofluorescence}

Day 1: the presence of fibrin on the explants as demonstrated by fluorescein tagged anti-fibrin antibody was noted until day 16 (Figure 3a). This was predominantly on the under surface of the explant. By day 16 , this positivity had disappeared, indicating that the fibrin glue had dissolved (Figure 3b).

\section{Discussion}

Fibrin glue consists of fibrinogen and thrombin, both of which are essential factors in the coagulation pathway. Thrombin is a serine protease that converts fibrinogen into insoluble strands of fibrin. Fibrin itself is a protein

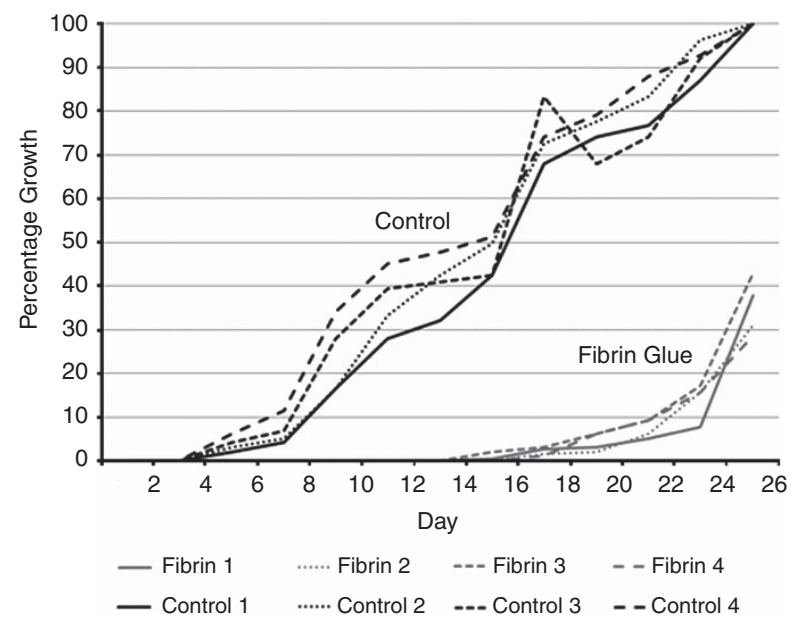

Figure 2 Graphic representation of percentage cell growth (y axis) between explants attached with fibrin glue and controls (no glue) plotted over time in days ( $x$ axis). 

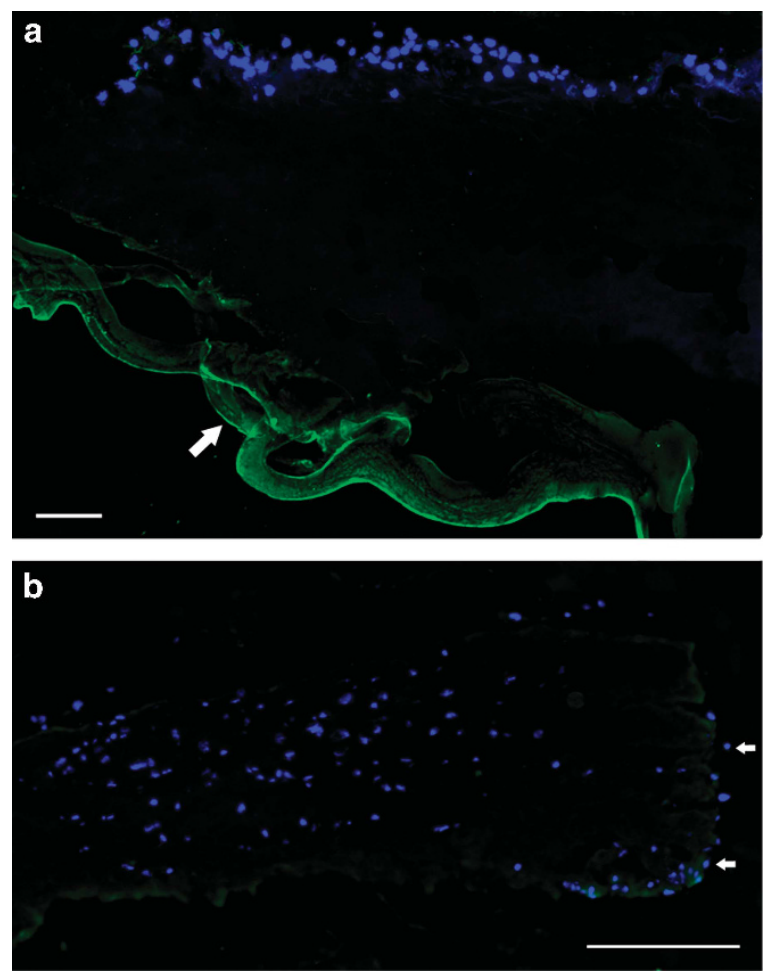

Figure 3 (a) Day 1 fibrin $(\times 40)$ : fibrin glue present (white arrow). Anti-fibrin (green) and DAPI (blue) nuclei counterstain (scale bar $=100 \mu \mathrm{m}$ ). (b) Day 16 fibrin $(\times 40)$ : fibrin glue has dissolved and epithelial cell migration can be seen (white arrows) down the side of the explants (scale bar $=100 \mu \mathrm{m}$ ).

that is polymerised via a pathway involving multiple factors ${ }^{12-14}$ to form a 'net' that becomes a haemostatic clot and permits tissue adhesion. The commercially available fibrin glue, Tisseel, used in this study is dispensed in two paired syringes. One contains Tisseel solution (Tisseel Powder (Fibrinogen, XIII, and Fibronectin) and Aprotinin) and the other contains Thrombin solution (Thrombin Powder and Calcium Chloride). Aprotinin has anti-fibrinolytic properties that maintain the clot for $\sim 2$ weeks by delaying fibrinolysis, thereby enhancing the length of its affect. Physiological fibrinolysis occurs in 45 days in response to tissue fibrinolytic enzymes. ${ }^{15}$ The addition of aprotinin allows satisfactory clinical applications of fibrin glue by delaying fibrinolysis. ${ }^{16}$ It was interesting to note that even in vitro, in our experiments, fibrinolysis occurred by days 14-16 that is comparable to what happens in vivo. Observations from this study would therefore be relevant to in vivo applications.

Fibrin glue has recently become popular in ophthalmic surgery. There have been varying reports documenting the benefits of fibrin glue. The gold standard procedure for pterygium surgery is excision of the lesion with simultaneous autologous conjunctival graft. Fibrin glue has been documented to reduce the recurrence rate and reduce postoperative discomfort compared with sutures. One possible mechanism is that fibrin glue facilitates greater and prolonged adhesion of the graft with the underlying tissues and permits early graft vascularisation. ${ }^{17}$

The success of conjunctival autografts may be affected by conjunctival inflammation. Sutures induce a localised inflammatory response by causing trauma and can act as a nidus for infection. ${ }^{18}$ Eliminating sutures can be advantageous postoperatively, as they can cause irritation and in turn inflammation. In addition, sutures can provide a site where infection can harbour and may stimulate cornea neovascularisation.

Corneal perforations can be treated using fibrin glue to effectively plug the defect ${ }^{19}$ and fibrin glue has been reported to be effective in reducing lamellar keratoplasty surgery times in rabbit eyes with $71 \%$ of grafts remaining in place securely. ${ }^{20}$

Fibrin glue use in limbal stem cell transplantation from limbal allografts, ${ }^{6}$ cadaveric corneoscleral rims, ${ }^{5}$ and limbal explants with amniotic membrane grafts ${ }^{21}$ have all been reported to successfully secure grafts to the recipient site with no reported graft rejection or dislocation. However, as stated in the introduction, we noted that migration of epithelial cells from the explant was delayed when fibrin glue was used to attach the grafts. This study confirms that this is indeed the case and suggests that the effect is very likely to be a physical barrier effect of the fibrin glue. The initial delay corresponded to the time interval it took for the fibrin glue to dissolve. Thereafter, cell migration started and the rate of migration soon caught up with that depicted by the control explants. Thus, although use of fibrin glue will secure attachment without sutures, the glue will also encase the explant and prevent cells from migrating off the explant. Epithelial cell movement occurs by a process of 'sliding', 'migration', and 'proliferation'. The cells initially slide to cover a larger surface area, then cell membrane extensions in the form of lamellopodia and filopodia reach beyond the cell boundary, attach to the substrate, and draw the cell cytoplasm towards the points of attachment, resulting in cell migration. ${ }^{22}$ Cell proliferation complements these processes leading to increase in cell numbers required to cover large defects. Although all of the above are active processes, fibrin glue is unlikely to retard cell movement by actively interfering with any of these but rather by acting as a physical barrier to cell sliding and migration. This was evident in the observation that cell migration remained arrested until the fibrin wrapped around the explants started to undergo dissolution as demonstrated by immunofluorescent staining. Moreover, fibrin as a substrate is conducive to supporting cell migration and attachment as is evident in the well-established clinical 
practice of ex vivo expansion of ocular surface cells before transplantation of such sheets for ocular surface reconstruction propounded by Pellegrini et al. ${ }^{23,24}$

It has been demonstrated that the average time for initial cell growth from a limbal explant is $4.5 \pm 1.8$ days $^{25}$ and this is confirmed in this study. Following limbal stem cell transplantation, it is crucial for the surface to establish a rapid and complete corneal epithelial cover. However, especially in cases of autolimbal and living related limbal grafts, where only two explants of approximately two clock hours each are used, the conjunctival epithelium competes against explant-derived corneal epithelium to cover the surface. This necessitates the use of sequential sector conjunctival epitheliectomy to keep the conjunctival epithelium at bay by repeated 'scraping' or 'brushing' of the conjunctival cells. ${ }^{26}$ The barrier effect of fibrin in containing migration of cells can be exploited in this context by using fibrin glue to attach the edges of the recessed conjunctiva to the episclera/ sclera, using fibrin in excess to cover the edges of the recessed conjunctiva. This can be complemented by avoiding use of fibrin to attach the limbal explants. This approach should delay conjunctival epithelial migration and allow more time for explant-derived limbal cells to cover the corneal surface, thus avoiding or reducing the number of times SSCE may be required. Although we did not directly test the migration of cells from conjunctival sheets in vitro, it is likely that fibrin would have the same physical barrier effects on conjunctival cells as well. Furthermore, we have used fibrin in this context in vivo during ocular surface reconstruction and observed the beneficial effect of fibrin glue in retarding cell migration from the conjunctiva (personal observation, Dua HS, 2015).

Experiments on cell culture have demonstrated a beneficial effect of fibrin coating of the substrate on cell migration. Epithelial cells migrate and adhere better on plastic coated with fibrin. Deshpande et $a l^{27}$ have recently reported favourable results of limbal epithelial cell growth on a biodegradable synthetic membrane composed of poly (D,L-lactide-co-glycolide) and coated with fibrin. However, they had spread a thin film of fibrin glue to allow for cell adhesion. This observation is not in contradiction to our results. When fibrin glue is used to secure the explant, inevitably some amount covers the sides of the explant, preventing cells to grow through the fibrin and, as explained above, this fact can be exploited to clinical advantage when it is desirable to retard cell migration.

In summary, based on our in vitro study, we propose that during limbal stem cell transplantation, fibrin glue can be used to introduce a potential temporary barrier to reduce conjunctival epithelial cells from competitively migrating across the corneal stroma. This then would favour corneal epithelial cell growth from the transplanted explants and can assist in achieving corneal epithelialisation with the correct phenotype of limbusderived epithelial cells. Conversely, the use of fibrin glue to secure the limbal explants should be considered with caution, and when contemplated, a very thin layer should be applied ensuring that it does not extend to the edges of the explant(s).

\section{Summary}

\section{What was known before}

- Fibrin glue has been used successfully in numerous ophthalmic surgical procedures. Recently, fibrin glue has been used in limbal stem cell transplantation to reduce both operative time and to negate the need for sutures.

\section{What this study adds}

- Fibrin glue acts as a physical barrier to epithelial cell migration and can potentially interfere with explantderived limbal epithelial cell migration on to the corneal surface. We propose that glue should be used to attach the conjunctival frill of the limbal explant but not the explant itself. However, it can be used to attach the recessed peritomised conjunctiva and delay conjunctival cell migration, which is desirable.

\section{Conflict of interest}

The authors declare no conflict of interest.

\section{Acknowledgements}

This study was funded by the Elizabeth C King Trust, Pittsburgh, USA.

\section{References}

1 Dua HS, Joseph A, Shanmuganathan VA, Jones RE. Stem cell differentiation and the effects of deficiency. Eye 2003; 17: 877-885.

2 Dua HS, Saini JS, Azuara-Blanco A, Gupta P. Limbal stem cell deficiency: concept, aetiology, clinical presentation, diagnosis and management. Indian J Ophthalmol 2000; 48: 83-92.

3 Dua HS, Gomes JA, Singh A. Corneal epithelial wound healing. Br J Ophthalmol 1994; 78: 401-408.

4 Holland EJ. Epithelial transplantation for the management of severe ocular surface disease. Trans Am Ophthalmol Soc 1996; 94: 677-743.

5 Nassiri N, Pandya HK, Djalilian AR. Limbal allograft transplantation using fibrin glue. Arch Ophthalmol 2011; 129: 218-222.

6 Sonmez B, Beden U. Fibrin glue-assisted sutureless limbal stem cell transplantation surgery for the treatment of severe ocular chemical injury. Cornea 2011; 30: 296-300.

7 Welder JD, Pandya HK, Nassiri N, Djalilian AR. Conjunctival limbal autograft and allograft transplantation using fibrin glue. Ophthalmic Surg Lasers Imaging 2012; 43: 323-327.

8 Sangwan VS, Basu S, MacNeil S, Balasubramanian D. Simple limbal epithelial transplantation (SLET): a novel surgical 
technique for the treatment of unilateral limbal stem cell deficiency. Br J Ophthalmol 2012; 96: 931-934.

9 Ozdamar Y, Mutevelli S, Han U, Ileri D, Onal B, Ilhan O et al. A comparative study of tissue glue and vicryl suture for closing limbal-conjunctival autografts and histologic evaluation after pterygium excision. Cornea 2008; 27: 552-558.

10 Srinivasan S, Dollin M, McAllum P, Berger Y, Rootman DS, Slomovic AR. Fibrin glue versus sutures for attaching the conjunctival autograft in pterygium surgery: a prospective observer masked clinical trial. Br J Ophthalmol 2009; 93: 215-218.

11 Uy HS, Reyes JM, Flores JD, Lim-Bon-Siong R. Comparison of fibrin glue and sutures for attaching conjunctival autografts after pterygium excision. Ophthalmology 2005; 112: 667-671.

12 Chung SI. Comparative studies on tissue transglutaminase and factor XIII. Ann NY Acad Sci 1972; 202: 240-255.

13 Iwanaga S, Suzuki K, Hashimoto S. Bovine plasma coldinsoluble globulin: gross structure and function. Ann NY Acad Sci 1978; 312: 56-73.

14 Sakata Y, Aoki N. Significance of cross-linking of alpha 2-plasmin inhibitor to fibrin in inhibition of fibrinolysis and in hemostasis. J Clin Invest 1982; 69: 536-542.

15 Gharaibeh A, Savage HI, Scherer RW, Goldberg MF, Lindsley K. Medical interventions for traumatic hyphema. Cochrane Database Syst Rev 2011; (1): CD005431.

16 Haberland G, McConn R. A rationale for the therapeutic action of aprotinin. Fed Proc 1979; 38: 2760-2767.

17 Koranyi G, Seregard S, Kopp ED. The cut-and-paste method for primary pterygium surgery: long-term follow-up. Acta Ophthalmol Scand 2005; 83: 298-301.

18 Sridhar MS, Bansal AK, Rao GN. Surgically induced necrotizing scleritis after pterygium excision and conjunctival autograft. Cornea 2002; 21: 305-307.
19 Weiss JL, Williams P, Lindstrom RL, Doughman DJ. The use of tissue adhesive in corneal perforations. Ophthalmology 1983; 90: 610-615.

20 Kim MS, Kim JH. Effects of tissue adhesive (Tisseel) on corneal wound healing in lamellar keratoplasty in rabbits. Korean J Ophthalmol 1989; 3: 14-21.

21 Zakaria N, Koppen C, Van Tendeloo V, Berneman Z, Hopkinson A, Tassignon MJ. Standardized limbal epithelial stem cell graft generation and transplantation. Tissue Eng Part C Methods 2010; 16: 921-927.

22 Dua HS, Forrester JV. Clinical patterns of corneal epithelial wound healing. Am J Ophthalmol 1987; 104: 481-489.

23 Pellegrini G, Ranno R, Stracuzzi G, Bondanza S, Guerra L, Zambruno $G$ et al. The control of epidermal stem cells (holoclones) in the treatment of massive full-thickness burns with autologous keratinocytes cultured on fibrin. Transplantation 1999; 68: 868-879.

24 Rama P, Bonini S, Lambiase A, Golisano O, Paterna P, De Luca $\mathrm{M}$ et al. Autologous fibrin-cultured limbal stem cells permanently restore the corneal surface of patients with total limbal stem cell deficiency. Transplantation 2001; 72: 1478-1485.

25 Kim HS, Jun Song X, de Paiva CS, Chen Z, Pflugfelder SC, Li DQ. Phenotypic characterization of human corneal epithelial cells expanded ex vivo from limbal explant and single cell cultures. Exp Eye Res 2004; 79: 41-49.

26 Dua HS. The conjunctiva in corneal epithelial wound healing. Br J Ophthalmol 1998; 82: 1407-1411.

27 Deshpande P, Ramachandran C, Sefat F, Mariappan I, Johnson C, McKean R et al. Simplifying corneal surface regeneration using a biodegradable synthetic membrane and limbal tissue explants. Biomaterials 2013; 34: 5088-5106. 